\title{
On-Line Booking Policies and Competitive Analysis of Medical Examination in Hospital
}

\author{
Li Luo, ${ }^{1}$ Chun-rong Qin, ${ }^{1}$ Shi-jun Tang, ${ }^{1}$ Xian Chen, ${ }^{2}$ and Hui-li Guo ${ }^{2}$ \\ ${ }^{1}$ Service Management Institute of Business School, Sichuan University, Chengdu 610064, China \\ ${ }^{2}$ The West China Hospital, Sichuan University, Chengdu 610064, China \\ Correspondence should be addressed to Chun-rong Qin; jianghu525@126.com
}

Received 8 March 2014; Accepted 17 April 2014; Published 7 May 2014

Academic Editor: Jian-Wen Peng

Copyright (c) $2014 \mathrm{Li} \mathrm{Luo} \mathrm{et} \mathrm{al.} \mathrm{This} \mathrm{is} \mathrm{an} \mathrm{open} \mathrm{access} \mathrm{article} \mathrm{distributed} \mathrm{under} \mathrm{the} \mathrm{Creative} \mathrm{Commons} \mathrm{Attribution} \mathrm{License,} \mathrm{which}$ permits unrestricted use, distribution, and reproduction in any medium, provided the original work is properly cited.

\begin{abstract}
From the on-line point, we consider the hospital's medical examination appointment problem with hierarchical machines. This approach eliminates the need for both demand forecasts and a risk-neutrality assumption. Due to different unit revenue, uncertain demand, and arrival of patients, we design on-line booking policies for two kinds of different situations from the perspective of online policy and competitive analysis. After that, we prove the optimal competitive ratios. Through numerical examples, we compare advantages and disadvantages between on-line policies and traditional policies, finding that there is different superiority for these two policies under different arrival sequences.
\end{abstract}

\section{Introduction}

In this paper, we investigate an on-line hierarchical scheduling problem on medical equipment, such as CT scanning. In hospital, patients arrive one by one randomly and each may be scheduled only on a certain machine. When a patient is assigned to a machine, it is not permitted to be manmodified. Namely, each patient has a set of available devices and a corresponding hierarchy. Each device has a patients set and a corresponding hierarchy. Patients cannot be scheduled on a device with lower hierarchy and the devices can only examine patients with no higher hierarchy. This constraint reflects different service capabilities of the devices, while it is very common in the practice (such as the customer level in services and the server level in communications). These issues can be summarized as hierarchical scheduling problem.

Suppose the service capacity of each device is $N$ in a given cycle, the service times of each patient are the same but the revenue is different. The goal of this study is to examine as many patients as possible to maximize hospitals' social revenue with successful appointments. Because it is unknown in advance how many patients will be present and which hierarchies they belong to, this social revenue-maximization problem is an on-line fashion.
This problem is motivated by practical cases in the Radiology Department of the West China Hospital. In this department, the CT scanning divides into two types depending on the complexity of the examinations, enhanced CT scanning and regular CT scanning. There are two CTs; CT1 is used for doing the regular scanning while CT2 is usually for enhanced scanning. The examination time of regular scanning is 1-2 minutes (excluding the preparation time and adjustment time of examination, such as the time for position adjustment and breath adjustment). The enhanced scanning needs to inject contrast agent, test allergy, and enhancer. It is worth noting that enhancer is unique to timeliness, so the patients should complete the examination as soon as enhancer is injected; otherwise the efficacy will expire.

In the actual appointment, the regular scanning patients can book CT1 or CT2, while the enhanced scanning patients can only book CT2. Now the appointment mode of the West China Hospital is patients making appointments in the integrated service station during a period of half day. Patients arrive at the service station one by one (inpatients usually arrive in batch) to make an appointment; then physicians judge people as regular scanning patients or enhanced scanning patients by synthesizing all kinds of factors. After that, nurses arrange the regular scanning patients to CT1 
and the enhanced scanning patients to CT2. When the capacity of CT1 is lacking, nurses will arrange patients on the first-come-first-served basis and transfer surplus patients to CT2. The obvious disadvantage of this mode is that it does not consider the different unit revenue from two types of patients. In general, the unit revenue from regular scanning patients is lower than the revenue from enhanced scanning patients. Certainly we cannot measure patients' value from the perspective of revenue, and that is why the hospitals choose the first-come-first-served as the basic rule. But from the social return, serving an enhanced scanning patient is also more important than serving a regular scanning patient. For CT2, if it accepts a regular scanning patient, it may reject a potential enhanced scanning patient; if it refuses the present regular scanning patient ("refuse" means patients have to be delayed to another day), it may be idle and loses this revenue. Therefore the service station should determine the number of regular scanning patients in the CT2 to generate better returns. Our study considers this problem from the view of on-line booking and competitive analysis.

Currently, there is a lot of literature on hierarchical online scheduling, such as literature [1-6]. However, few scholars considered such social revenue maximization problem in hospital. Compared with the traditional literature, there are two differences. First, the capacity of the device is not limited in the traditional literature; all jobs must be machined. Second, the termination condition of traditional scheduling algorithm is that all jobs are completed, and the goal is usually to complete the processing of all jobs as soon as possible. The common optimization objectives are minimizing the total completion time $\left(\sum C_{j}\right)$ [7-9] or the maximum completion time $\left(C_{\max }\right)[10,11]$, while our work is to maximize the social return.

In this paper, we assume that the service capacity of each device in a certain period is $N$ and the service time of each patient is the same, but the unit revenue is different. Thus, the goal of this paper is to maximize the total social revenue from all accepted patients in certain period. Further, we assume that the patients and the devices are hierarchical. A patient can make a reservation of a device whose grade is not lower than the level of his own.

Next, considering the different unit revenue from different types of patients, the paper studies two-hierarchy on-line scheduling problem in two situations.

\section{On-Line Booking and Competitive Analysis}

Suppose there are two devices: $M_{1}$ and $M_{2}$. The grade of $M_{1}$ is 1 and that of $M_{2}$ is 2 . The same capacity of each device is $N$. Two types of patients are involved: type 1 and type 2 . The level of type 1 is 1 and that of type 2 is 2 . Namely, patients of type 1 can book $M_{1}$ and $M_{2}$, while patients of type 2 can only book $M_{2}$. Assume the service time of each patient is the same and the unit revenue is different. The unit revenue of examining each type 1 patient with $M_{1}$ is $r_{1}$ while it is $r_{1}^{\prime}$ with $M_{2}$, and the unit revenue of examining each type 2 patient with $M_{2}$ is $r_{2}$. Obviously, $r_{1} \geq r_{1}^{\prime}$; because the cost of examining the patients of type 1 with $M_{2}$ is higher than examining them with $M_{1}$, there will be lower social income. So it is impossible for the hospital manager to arrange patients of type 1 to $M_{2}$ when $M_{1}$ has reservation capability. As for $r_{1}^{\prime}$ and $r_{2}$, which is higher is uncertain, so we discuss the on-line problem from two perspectives.

2.1. $r_{1}^{\prime} \leq r_{2}$. In this situation, the unit revenue of using $M_{2}$ to examine type 2 patients is higher than to examine type 1 patients. The manager should decide whether to arrange type 1 patients to $M_{2}$ or not. On the one hand, the service capacity of $M_{1}$ is insufficient, and the number of type 1 patients is much larger than that of type 2 patients; it is better to transfer some patients of type 1 to $M_{2}$. On the other hand, if there are plenty of type 2 patients, only few type 1 patients can be arranged to $M_{2}$ while enough capacity should be kept for patients of type 2 .

This paper designs an optimal on-line policy ST1; moreover, the competitive ratio is $c=r_{2}\left(r_{1}+r_{1}^{\prime}\right) /\left(2 r_{1}^{\prime} r_{2}+r_{1} r_{2}-r_{1}^{\prime 2}\right)$.

Suppose $n_{1}$ is the number of type 1 patients who are arranged to $M_{1}, n_{2}$ is the number of type 1 and type 2 patients who use $M_{2}$, and $n_{2}^{1}$ is the number of type 1 patients with $M_{2}$. The on-line policy is as follow.

ST1 Policy. (1) Suppose $\tilde{n}_{1}$ is the total number of being accepted type 1 patients by $M_{1}$ before the next arriving type 1 patient, $\widetilde{n}_{2}$ is the total number of being accepted type 1 and type 2 patients by $M_{2}$ before the next arriving patient, $\tilde{n}_{2}^{1}$ is the total number of being accepted type 1 patients by $M_{2}$ before the next arriving type 1 patient. At the beginning of the appointment time, $\widetilde{n}_{1}=0, \widetilde{n}_{2}=0$, and $\tilde{n}_{2}^{1}=0$.

(2) For the next arriving patient, consider the following.

$\left(\mathrm{A}_{1}\right)$ The patient type is type 1 . If $\tilde{n}_{1}<N$, arrange the patient to $M_{1}$. If $\tilde{n}_{1}=N, \tilde{n}_{2}<N$, and $\tilde{n}_{2}^{1}<y_{1} N$ (where $\left.y_{1}=r_{1}^{\prime}\left(r_{1}+r_{2}\right) /\left(2 r_{1}^{\prime} r_{2}+r_{1} r_{2}-r_{1}^{\prime 2}\right)\right)$, arrange the patient to $M_{2}$. Otherwise, refuse.

$\left(\mathrm{A}_{2}\right)$ The patient type is type 2 . If $\tilde{n}_{2}<N$, arrange the patient to $M_{2}$. Otherwise, refuse.

Theorem 1. The competitive ratio of ST1 policy is $c_{1}=r_{2}\left(r_{1}+\right.$ $\left.r_{1}^{\prime}\right) /\left(2 r_{1}^{\prime} r_{2}+r_{1} r_{2}-r_{1}^{\prime 2}\right)$.

Proof. For any input sequence $I, V^{\prime}(I)$ is the total social revenue of ST1 policy and $V^{*}(I)$ is the total social revenue of the optimal off-line policy. Consider the following four circumstances.

(1) $n_{1}<N$. In this case, for any input sequence $I$, online algorithm ST1 is the same as the optimal off-line algorithm; both of them will arrange all patients of type 1 to $M_{1}$ and all patients of type 2 to $M_{2}$. So, $V^{\prime}(I)=V^{*}(I)$; namely, $V^{\prime}(I) / V^{*}(I)=1$. 
(2) $n_{1}=N, n_{2}=N$. At the moment, the total social revenue of ST1 policy is

$$
\begin{aligned}
V^{\prime}(I) & =r_{1} N+r_{1}^{\prime} n_{2}^{1}+r_{2}\left(N-n_{2}^{1}\right) \\
& \geq r_{1} N+r_{1}^{\prime} y_{1} N+r_{2}\left(N-y_{1} N\right) \\
& =r_{1} N+r_{1}^{\prime} y_{1} N+r_{2}\left(1-y_{1}\right) N .
\end{aligned}
$$

The optimal off-line revenue is $V^{*}(I) \leq r_{1} N+r_{2} N$; then

$$
\begin{aligned}
\frac{V^{\prime}(I)}{V^{*}(I)} & \geq \frac{r_{1} N+r_{1}^{\prime} n_{2}^{1}+r_{2}\left(N-n_{2}^{1}\right)}{r_{1} N+r_{2} N} \\
& \geq \frac{r_{1} N+r_{1}^{\prime} y_{1} N+r_{2}\left(1-y_{1}\right) N}{r_{1} N+r_{2} N} \\
& =\frac{r_{1}+r_{1}^{\prime} y_{1}+r_{2}\left(1-y_{1}\right)}{r_{1}+r_{2}}=\frac{r_{2}\left(r_{1}+r_{1}^{\prime}\right)}{2 r_{1}^{\prime} r_{2}+r_{1} r_{2}-r_{1}^{\prime 2}} .
\end{aligned}
$$

(3) $n_{1}=N, n_{2}<N$, and before the policy stops, there is always $n_{2}^{1}<y_{1} N$. By this time, all arrived demands can be accepted. So $V^{\prime}(I)=V^{*}(I)$; namely, $V^{\prime}(I) / V^{*}(I)=1$.

(4) $n_{1}=N, n_{2}<N$, and at some period before the policy stops, $n_{2}^{1}=y_{1} N$. In this case, off-line adversary will design a special input sequence: after $n_{2}^{1}=y_{1} N$, only type 1 patients will come. Under this kind of bad sequence, the total social revenue of ST1 policy is

$$
V^{\prime}(I)=r_{1} N+r_{1}^{\prime} y_{1} N+r_{2}\left(n_{2}-y_{1} N\right) .
$$

The total social revenue of the optimal off-line policy is

$V^{*}(I)=r_{1} N+r_{1}^{\prime}\left(N-\left(n_{2}-y_{1} N\right)\right)+r_{2}\left(n_{2}-y_{1} N\right)$.

Then

$$
\begin{aligned}
\frac{V^{\prime}(I)}{V^{*}(I)} & =\frac{r_{1} N+r_{1}^{\prime} y_{1} N+r_{2}\left(n_{2}-y_{1} N\right)}{r_{1} N+r_{1}^{\prime}\left(N-\left(n_{2}-y_{1} N\right)\right)+r_{2}\left(n_{2}-y_{1} N\right)} \\
& \geq \frac{r_{1} N+r_{1}^{\prime} y_{1} N+r_{2}\left(n_{2}-y_{1} N\right)}{r_{1} N+r_{1}^{\prime} N+r_{2}\left(n_{2}-y_{1} N\right)} \\
& \geq \frac{r_{1} N+r_{1}^{\prime} y_{1} N}{r_{1} N+r_{1}^{\prime} N}=\frac{r_{1}+r_{1}^{\prime} y_{1}}{r_{1}+r_{1}^{\prime}} \\
& =\frac{r_{2}\left(r_{1}+r_{1}^{\prime}\right)}{2 r_{1}^{\prime} r_{2}+r_{1} r_{2}-r_{1}^{\prime 2}} .
\end{aligned}
$$

To sum up, for any input sequence $I$, there is always

$$
V(I) \geq \frac{r_{2}\left(r_{1}+r_{1}^{\prime}\right)}{2 r_{1}^{\prime} r_{2}+r_{1} r_{2}-r_{1}^{\prime 2}} V^{*}(I) .
$$

Then the competitive ratio of ST1 policy is $c_{1}=r_{2}\left(r_{1}+\right.$ $\left.r_{1}^{\prime}\right) /\left(2 r_{1}^{\prime} r_{2}+r_{1} r_{2}-r_{1}^{\prime 2}\right)$.
Next, we will prove that ST1 algorithm is the optimal online scheduling algorithm.

Theorem 2. ST1 algorithm is the optimal on-line scheduling algorithm.

Proof. In order to prove that ST1 algorithm is the optimal online scheduling algorithm, we only need to prove that, for any other on-line scheduling algorithm, its competitive ratio is not more than $r_{2}\left(r_{1}+r_{1}^{\prime}\right) /\left(2 r_{1}^{\prime} r_{2}+r_{1} r_{2}-r_{1}^{\prime 2}\right)$.

Consider the following two special arrival sequences $I_{1}$ and $I_{2}$. In $I_{1}$, there first come $2 N$ type 1 patients, and then come $N$ type 2 patients. In $I_{2}$, there first come $2 N$ type 1 patients, and then have no type 2 patients to come. At the moment, the total revenue of optimal off-line policy is

$$
V^{*}\left(I_{1}\right)=r_{1} N+r_{2} N, \quad V^{*}\left(I_{2}\right)=r_{1} N+r_{1}^{\prime} N .
$$

For any on-line scheduling algorithm $A$, let $x \mathrm{~N}$ be the total number of type 1 patients that can be accepted by $M_{2}$. Obviously, $0 \leq x \leq 1$. Accordingly,

$$
\begin{aligned}
& V\left(I_{1}\right)=r_{1} N+r_{1}^{\prime} x N+r_{2}(N-x N), \\
& V\left(I_{2}\right)=r_{1} N+r_{1}^{\prime} x N .
\end{aligned}
$$

By calculating, we find the following.

When

$$
\begin{gathered}
x \geq y_{1}=\frac{\left(r_{1}+r_{2}\right) r_{1}^{\prime}}{2 r_{1}^{\prime} r_{2}-r_{1}^{\prime 2}+r_{1} r_{2}}, \\
V\left(I_{1}\right) \leq \frac{r_{2}\left(r_{1}+r_{1}^{\prime}\right)}{2 r_{1}^{\prime} r_{2}+r_{1} r_{2}-r_{1}^{\prime 2}} V^{*}\left(I_{1}\right) .
\end{gathered}
$$

When

$$
\begin{gathered}
x \leq y_{1}=\frac{\left(r_{1}+r_{2}\right) r_{1}^{\prime}}{2 r_{1}^{\prime} r_{2}-r_{1}^{\prime 2}+r_{1} r_{2}}, \\
V\left(I_{2}\right) \leq \frac{r_{2}\left(r_{1}+r_{1}^{\prime}\right)}{2 r_{1}^{\prime} r_{2}+r_{1} r_{2}-r_{1}^{\prime 2}} V^{*}\left(I_{2}\right) .
\end{gathered}
$$

Then, for any on-line scheduling algorithm $A$, there exists some arrival sequence, whose total on-line revenue is not more than $r_{2}\left(r_{1}+r_{1}^{\prime}\right) /\left(2 r_{1}^{\prime} r_{2}+r_{1} r_{2}-r_{1}^{\prime 2}\right)$ times for the total optimal off-line revenue. Namely, for any on-line scheduling algorithm $A$, its competitive ratio is not more than $r_{2}\left(r_{1}+\right.$ $\left.r_{1}^{\prime}\right) /\left(2 r_{1}^{\prime} r_{2}+r_{1} r_{2}-r_{1}^{\prime 2}\right)$.

Combined with Theorem 1, we can see that ST1 algorithm is the optimal on-line scheduling algorithm; its competitive ratio is $r_{2}\left(r_{1}+r_{1}^{\prime}\right) /\left(2 r_{1}^{\prime} r_{2}+r_{1} r_{2}-r_{1}^{\prime 2}\right)$.

2.2. $r_{1}^{\prime}>r_{2}$. In this condition, the unit revenue of using $M_{2}$ to examine type 1 patients is higher than to examine type 2 patients. The manager should decide whether to accept the request from type 2 patients. If the service capacity of $M_{1}$ is sufficient and the amount of type 2 patients far exceeds that 
TABLE 1: Comparison of two strategies in Case 1.

\begin{tabular}{|c|c|c|c|c|c|c|c|c|c|c|c|}
\hline & & \multicolumn{10}{|c|}{ Patient number } \\
\hline & & 1 & 2 & 3 & 4 & 5 & 6 & 7 & 8 & 9 & 10 \\
\hline & Patient type & 1 & 1 & 1 & 1 & 1 & 1 & 1 & 1 & 1 & 2 \\
\hline \multirow{4}{*}{ Device } & ST1 policy (total revenue: 4850) & 1 & 1 & 1 & 1 & 1 & 1 & 1 & 1 & 1 & 2 \\
\hline & Traditional policy (total revenue: 5450) & 1 & 1 & 1 & 1 & 1 & 1 & 1 & 1 & 1 & 2 \\
\hline & & \multicolumn{10}{|c|}{ Patient number } \\
\hline & & 11 & 12 & 13 & 14 & 15 & 16 & 17 & 18 & 19 & 20 \\
\hline \multirow{5}{*}{ Device } & Patient type & 1 & 1 & 1 & 1 & 1 & 1 & 2 & 2 & 1 & 1 \\
\hline & ST1 policy (total revenue: 4850 ) & 1 & 1 & 1 & 1 & 1 & 1 & 2 & 2 & 1 & 1 \\
\hline & Traditional policy (total revenue: 5450) & 1 & 1 & 1 & 1 & 1 & 1 & 2 & 2 & 1 & 1 \\
\hline & & \multicolumn{10}{|c|}{ Patient number } \\
\hline & & 21 & 22 & 23 & 24 & 25 & 26 & 27 & 28 & 29 & 30 \\
\hline \multirow{5}{*}{ Device } & Patient type & 1 & 1 & 1 & 1 & 1 & 1 & 1 & 1 & 1 & 1 \\
\hline & ST1 policy (total revenue: 4850 ) & 1 & 1 & 1 & 2 & 2 & 2 & 2 & 2 & 2 & 2 \\
\hline & Traditional policy (total revenue: 5450) & 1 & 1 & 1 & 2 & 2 & 2 & 2 & 2 & 2 & 2 \\
\hline & & \multicolumn{10}{|c|}{ Patient number } \\
\hline & & 31 & 32 & 33 & 34 & 35 & 36 & 37 & 38 & 39 & 40 \\
\hline \multirow{3}{*}{ Device } & Patient type & 1 & 1 & 1 & 1 & 1 & 1 & 1 & 1 & 1 & 1 \\
\hline & ST1 policy (total revenue: 4850) & 2 & 2 & 2 & 2 & 0 & 0 & 0 & 0 & 0 & 0 \\
\hline & Traditional policy (total revenue: 5450) & 2 & 2 & 2 & 2 & 2 & 2 & 2 & 2 & 2 & 2 \\
\hline
\end{tabular}

of type 1 patients, it is better to accept a part of requests from type 2 patients. On the other hand, if there will be lots of type 1 patients, the manager cannot respond too much requests from type 2 patients and keep enough capacity of $M_{2}$ for subsequent patients of type 1 .

Next, we design an optimal on-line policy ST2; moreover, the competitive ratio is $c=r_{1}^{\prime}\left(r_{1}+r_{2}\right) /\left(2 r_{1}^{\prime} r_{2}+r_{1} r_{1}^{\prime}-r_{2}^{2}\right)$.

Suppose $n_{1}$ is the number of patients of type 1 who are arranged to $M_{1}, n_{2}$ is the number of type 1 and type 2 patients who use $M_{2}$, and $n_{2}^{2}$ is the number of type 2 patients with $M_{2}$. The on-line policy is as follow.

ST2 Policy. (1) Suppose $\widetilde{n}_{1}$ is the total number of being accepted type 1 patients by $M_{1}$ before the next arriving type 1 patient, $\widetilde{n}_{2}$ is the total number of being accepted type 1 and type 2 patients by $M_{2}$ before the next arriving patient, $\widetilde{n}_{2}^{2}$ is the total number of being accepted type 2 patients by $M_{2}$ before the next arriving type 2 patient. At the beginning of the appointment time, $\widetilde{n}_{1}=0, \widetilde{n}_{2}=0$, and $\widetilde{n}_{2}^{2}=0$.

(2) For the next arriving patient, consider the following.

$\left(\mathrm{B}_{1}\right)$ The patient type is type 1 . If $\tilde{n}_{1}<N$, arrange the patient to $M_{1}$. If $\widetilde{n}_{1}=N$ and $\widetilde{n}_{2}<N$, arrange the patient to $M_{2}$. Otherwise, refuse.

$\left(\mathrm{B}_{2}\right)$ The patient type is type 2. If $\widetilde{n}_{2}<N$ and $\widetilde{n}_{2}^{2}<y_{2} N$ (where $\left.y_{2}=r_{2}\left(r_{1}+r_{1}^{\prime}\right) /\left(2 r_{1}^{\prime} r_{2}+r_{1} r_{1}^{\prime}-r_{2}^{2}\right)\right)$, arrange the patient to $M_{2}$. Otherwise, refuse.
Theorem 3. ST2 algorithm is the optimal on-line scheduling algorithm; its competitive ratio is $c_{2}=r_{1}^{\prime}\left(r_{1}+r_{2}\right) /\left(2 r_{1}^{\prime} r_{2}+r_{1} r_{1}^{\prime}-\right.$ $\left.r_{2}^{2}\right)$.

The proof of Theorem 3 is similar to the proof of Theorems 1 and 2, so it is not explained here.

Through the conclusions of three theorems above, we can find the difference between on-line policy and off-line policy when the service capacity of $M_{1}$ is insufficient. If the service capacity of $M_{1}$ is enough, all patients of type 1 will be arranged to $M_{1}$, not to $M_{2}$. When the service capacity of $M_{1}$ is not enough, the subsequent patients of type 1 could be arranged to $M_{2}$; there are two different on-line policies according to different unit revenue. For ST1 policy, considering the unit revenue of using $M_{2}$ to examine type 2 patients is higher than to examine type 1 patients, when $M_{2}$ has reservation capability, the manager should control the number of type 1 patients on $M_{2}$ and be sure the request of type 2 patients can be met as far as possible. For ST2 policy, considering the unit revenue of using $M_{2}$ to examine type 1 patients is higher than to examine type 2 patients, the manager should control the number of patients of type 2 on $M_{2}$ and make sure patients of type 1 can be serviced.

\section{Contrast with Traditional Reservation Policy}

For $r_{1}^{\prime}<r_{2}$, by calculating, we have $y_{1}=r_{1}^{\prime}\left(r_{1}+r_{2}\right) /\left(2 r_{1}^{\prime} r_{2}+\right.$ $\left.r_{1} r_{2}-r_{1}^{\prime 2}\right)<c_{1}=r_{2}\left(r_{1}+r_{1}^{\prime}\right) /\left(2 r_{1}^{\prime} r_{2}+r_{1} r_{2}-r_{1}^{\prime 2}\right)$. Obviously, $y_{1}$ is 
TABle 2: Comparison of two strategies in Case 2.

\begin{tabular}{|c|c|c|c|c|c|c|c|c|c|c|c|}
\hline & & \multicolumn{10}{|c|}{ Patient number } \\
\hline & & 1 & 2 & 3 & 4 & 5 & 6 & 7 & 8 & 9 & 10 \\
\hline & Patient type & 1 & 1 & 1 & 2 & 1 & 1 & 1 & 1 & 2 & 1 \\
\hline \multirow{4}{*}{ Device } & ST1 policy (total revenue: 6350 ) & 1 & 1 & 1 & 2 & 1 & 1 & 1 & 1 & 2 & 1 \\
\hline & Traditional policy (total revenue: 5450 ) & 1 & 1 & 1 & 2 & 1 & 1 & 1 & 1 & 2 & 1 \\
\hline & & \multicolumn{10}{|c|}{ Patient number } \\
\hline & & 11 & 12 & 13 & 14 & 15 & 16 & 17 & 18 & 19 & 20 \\
\hline \multirow{5}{*}{ Device } & Patient type & 1 & 1 & 1 & 1 & 1 & 1 & 1 & 1 & 1 & 1 \\
\hline & ST1 policy (total revenue: 6350 ) & 1 & 1 & 1 & 1 & 1 & 1 & 1 & 1 & 1 & 1 \\
\hline & Traditional policy (total revenue: 5450) & 1 & 1 & 1 & 1 & 1 & 1 & 1 & 1 & 1 & 1 \\
\hline & & \multicolumn{10}{|c|}{ Patient number } \\
\hline & & 21 & 22 & 23 & 24 & 25 & 26 & 27 & 28 & 29 & 30 \\
\hline \multirow{5}{*}{ Device } & Patient type & 1 & 1 & 1 & 1 & 1 & 1 & 1 & 1 & 1 & 1 \\
\hline & ST1 policy (total revenue: 6350) & 1 & 1 & 2 & 2 & 2 & 2 & 2 & 2 & 2 & 2 \\
\hline & Traditional policy (total revenue: 5450) & 1 & 1 & 2 & 2 & 2 & 2 & 2 & 2 & 2 & 2 \\
\hline & & \multicolumn{10}{|c|}{ Patient number } \\
\hline & & 31 & 32 & 33 & 34 & 35 & 36 & 37 & 38 & 39 & 40 \\
\hline \multirow{5}{*}{ Device } & Patient type & 1 & 1 & 1 & 1 & 1 & 1 & 1 & 1 & 1 & 2 \\
\hline & ST1 policy (total revenue: 6350) & 2 & 2 & 2 & 0 & 0 & 0 & 0 & $\boldsymbol{0}$ & $\mathbf{0}$ & 2 \\
\hline & Traditional policy (total revenue: 5450 ) & 2 & 2 & 2 & 2 & 2 & 2 & 2 & 2 & 2 & 2 \\
\hline & & \multicolumn{10}{|c|}{ Patient number } \\
\hline & & 41 & 42 & 43 & 44 & 45 & 46 & 47 & 48 & 49 & 50 \\
\hline & Patient type & 2 & 2 & 2 & 2 & 2 & 2 & 2 & 2 & 2 & 2 \\
\hline \multirow{2}{*}{ Device } & ST1 policy (total revenue: 6350) & 2 & 2 & 2 & 2 & 2 & 2 & 0 & 0 & 0 & 0 \\
\hline & Traditional policy (total revenue: 5450 ) & 0 & 0 & 0 & 0 & 0 & 0 & 0 & 0 & 0 & 0 \\
\hline
\end{tabular}

non-increasing in $r_{1}, r_{2}$ and is non-decreasing in $r_{1}^{\prime} . c_{1}$ is not decreasing with $r_{1}$ and is not increasing with $r_{2}$. However, it is not sure of $c_{1}=r_{2}\left(r_{1}+r_{1}^{\prime}\right) /\left(2 r_{1}^{\prime} r_{2}+r_{1} r_{2}-r_{1}^{\prime 2}\right)$ 's monotonicity about $r_{1}^{\prime}$, because

$$
\begin{aligned}
\frac{\partial c_{1}}{\partial r_{1}^{\prime}} & =\frac{r_{2}\left(2 r_{1}^{\prime} r_{2}+r_{1} r_{2}-r_{1}^{\prime 2}\right)-r_{2}\left(r_{1}+r_{1}^{\prime}\right)\left(2 r_{2}-2 r_{1}^{\prime}\right)}{\left(2 r_{1}^{\prime} r_{2}+r_{1} r_{2}-r_{1}^{\prime 2}\right)^{2}} \\
& =\frac{r_{2}\left(r_{1}^{\prime 2}-r_{1} r_{2}+2 r_{1} r_{1}^{\prime}\right)}{\left(2 r_{1}^{\prime} r_{2}+r_{1} r_{2}-r_{1}^{\prime 2}\right)^{2}} .
\end{aligned}
$$

When $r_{1}^{\prime} \in\left(0,-r_{1}+\sqrt{r_{1}^{2}+r_{1} r_{2}}\right), \partial c_{1} / \partial r_{1}^{\prime}<0$ and $c_{1}=$ $r_{2}\left(r_{1}+r_{1}^{\prime}\right) /\left(2 r_{1}^{\prime} r_{2}+r_{1} r_{2}-r_{1}^{\prime 2}\right)$ is non-increasing in $r_{1}^{\prime}$.

When $r_{1}^{\prime} \in\left[-r_{1}+\sqrt{r_{1}^{2}+r_{1} r_{2}}, r_{2}\right), \partial c_{1} / \partial r_{1}^{\prime} \geq 0$ and $c_{1}=$ $r_{2}\left(r_{1}+r_{1}^{\prime}\right) /\left(2 r_{1}^{\prime} r_{2}+r_{1} r_{2}-r_{1}^{\prime 2}\right)$ is non-decreasing in $r_{1}^{\prime}$.

In order to compare on-line booking in this paper with the traditional reservation (first-come-first-appointed), we consider the following two extreme cases.

Case 1. From the certain moment when the service capacity of $M_{1}$ is insufficient, the subsequent people are all type 1 patients.
Case 2. From the certain moment when the service capacity of $M_{1}$ is insufficient, some patients of type 2 arrive after patients of type 1 .

Consistent with the description above, we define $y_{1} N$ as the upper limit of type 1 patients on $M_{2}$. Suppose $N=20$, $r_{1}=150, r_{1}^{\prime}=100$, and $r_{2}=250$. In order to simplify the comparison process, suppose $\tilde{n}_{2}^{1}<y_{1} N$, where $\tilde{n}_{2}^{1}$ is the number of type 1 patients accepted by $M_{2}$. The analysis process is similar when $\tilde{n}_{2}^{1}=y_{1} N$. The revenue of on-line and traditional policy is shown in Tables 1 and 2 .

In Table 1, ST1 policy controls the accepted number of type 1 patients on $M_{2}$, and traditional reservation policy accepts appointments one by one according to the first-comefirst-appointed policy until the service capacity of $M_{2}$ runs out. So the revenue from traditional policy is higher than online policy.

In Table 2, ST1 policy refuses some patients of type 1 and keeps the capacity of $M_{2}$ for subsequent patients of type 2 who bring more yields. Traditional policy accepts the type 1 patients who come first so that the more valuable patients of type 2 are rejected by $M_{2}$.

The above comparison shows that two strategies exhibit different advantages according to different demand 
sequences. On-line policy in this paper is based on a worst case analysis and the actual arrival sequence is not always worst, so it is necessary to improve on-line revenue management policy design on the actual distribution of demand.

\section{Conclusion}

The medical technology appointment is core problem of hospital management. The nurses in service station face unknown demand sequences and should make a decision immediately when each patient arrives. On-line policy and competitive analysis are exactly suitable for decision problem with unknown demand sequences. This paper studies the revenue management problem of hospital medical examination according to the on-line policy. Based on the literature [12], we analyze the medical examination appointment model under different yield scenarios and design the optimal on-line booking policy. Finally, we compare the on-line policy with traditional reservation policy by numerical examples.

In the future, it is necessary to dynamically adjust the existing reservation policy based on demand information, because the demand sequence generally follows certain rules in practice. According to these laws, designing on-line policy with the latest achievements in competitive analysis will be a more realistic theoretical issue.

\section{Conflict of Interests}

The authors declare that there is no conflict of interests regarding the publication of this paper.

\section{Acknowledgments}

The authors would like to express their thanks to the referees for their valuable suggestions and comments. This work is supported by the National Natural Science Foundation of China (71131006, 71172197, and 70771068) and the Central University Fund of Sichuan University (skgt201202).

\section{References}

[1] A. Bar-Noy, A. Freund, and J. Naor, "On-line load balancing in a hierarchical server topology," SIAM Journal on Computing, vol. 31, no. 2, pp. 527-549, 2001.

[2] Y.-W. Jiang, Y. He, and C.-M. Tang, "Optimal online algorithms for scheduling on two identical machines under a grade of service," Journal of Zhejiang University: Science, vol. 7, no. 3, pp. 309-314, 2006.

[3] J. Park, S. Y. Chang, and K. Lee, "Online and semi-online scheduling of two machines under a grade of service provision," Operations Research Letters, vol. 34, no. 6, pp. 692-696, 2006.

[4] Y. Jiang, "Online scheduling on parallel machines with two GoS levels," Journal of Combinatorial Optimization, vol. 16, no. 1, pp. 28-38, 2008.

[5] A. Zhang, Y. Jiang, and Z. Tan, "Online parallel machines scheduling with two hierarchies," Theoretical Computer Science, vol. 410, no. 38-40, pp. 3597-3605, 2009.
[6] Z. Tan and A. Zhang, "A note on hierarchical scheduling on two uniform machines," Journal of Combinatorial Optimization, vol. 20, no. 1, pp. 85-95, 2010.

[7] C. Phillips, C. Stein, and J. Wein, "Scheduling jobs that arrive over time," in Proceedings of the 4th Workshop on Algorithms and Data Structures, pp. 86-97, 1995.

[8] J. A. Hoogeveen and A. P. A. Vestjens, "Optimal on-line algorithms for single-machine scheduling," in Proceedings of the 5th Integer Programming and Combinatorial Optimization Conference (IPCO '96), pp. 404-414, 1996.

[9] P. Liu and X. Lu, "On-line scheduling of parallel machines to minimize total completion times," Computers \& Operations Research, vol. 36, no. 9, pp. 2647-2652, 2009.

[10] U. Faigle, W. Kern, and G. Turán, "On the performance of online algorithms for partition problems," Acta Cybernetica, vol. 9, no. 2, pp. 107-119, 1989.

[11] B. Chen and A. P. A. Vestjens, "Scheduling on identical machines: how good is LPT in an on-line setting?" Operations Research Letters, vol. 21, no. 4, pp. 165-169, 1997.

[12] G.-Q. Ni and Y.-F. Xu, "Competitive analysis of dynamic online booking policies in revenue management," System Engineering Theory and Practice, vol. 31, no. 12, pp. 2308-2315, 2011 (Chinese). 


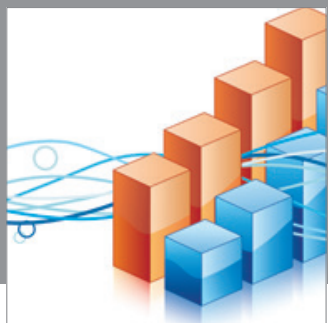

Advances in

Operations Research

mansans

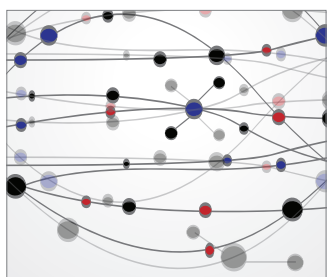

The Scientific World Journal
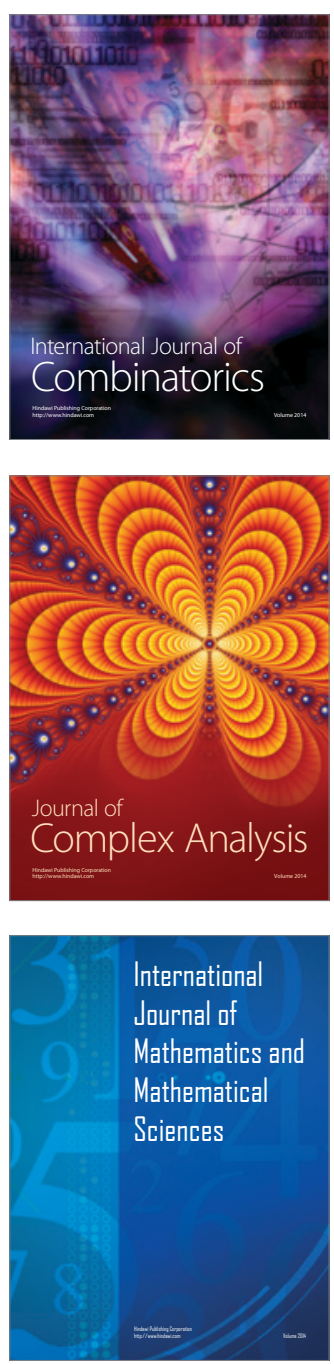
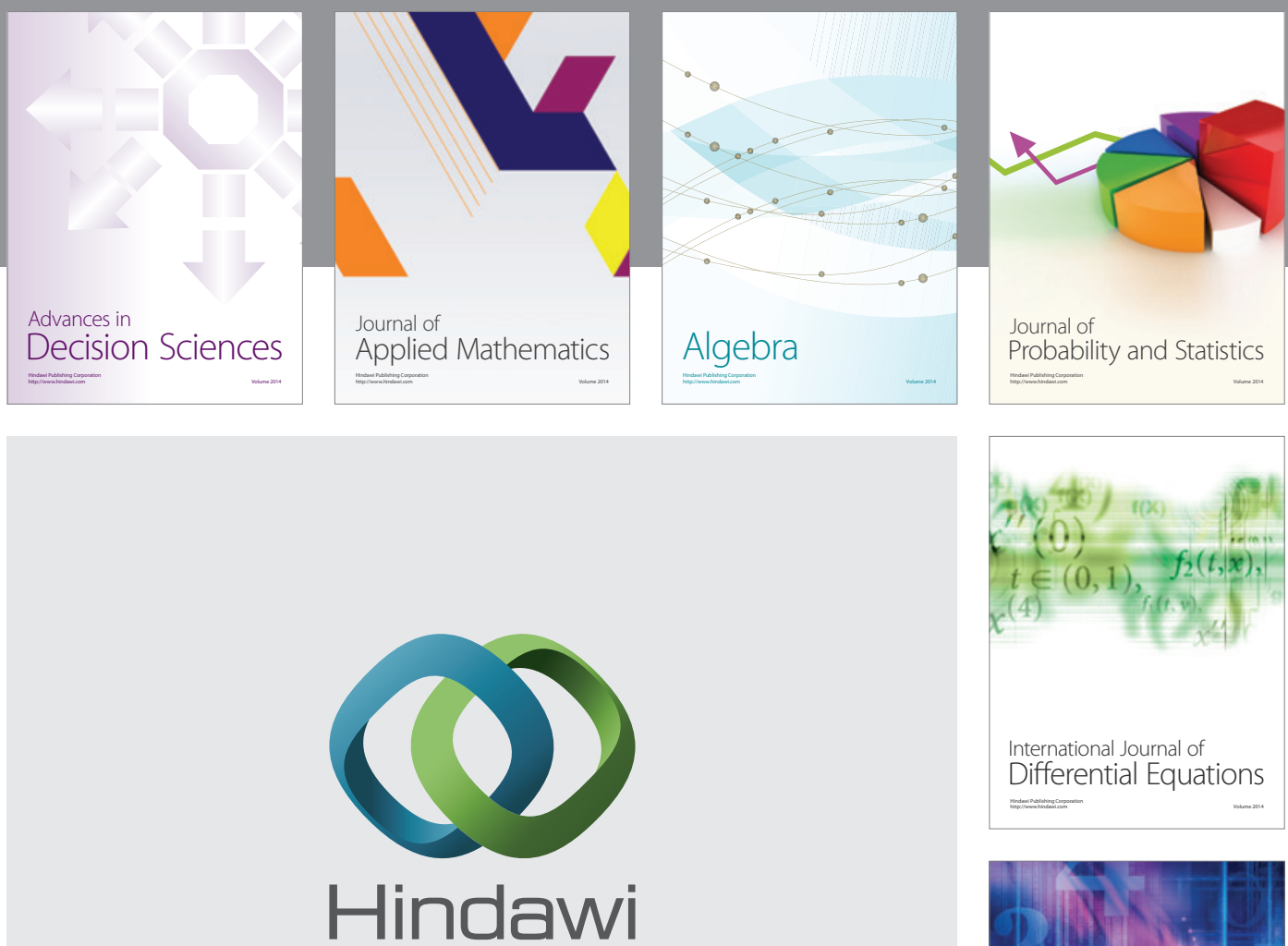

Submit your manuscripts at http://www.hindawi.com
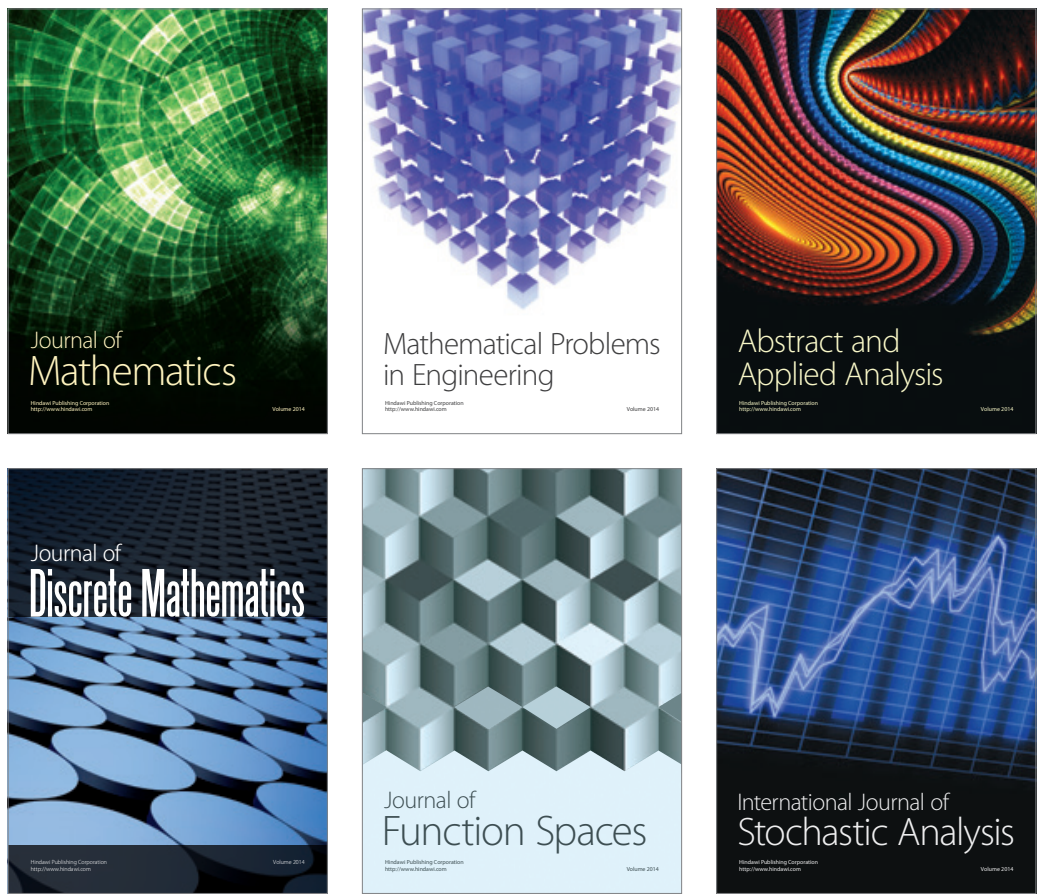

Journal of

Function Spaces

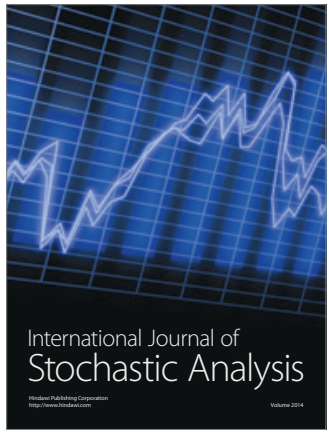

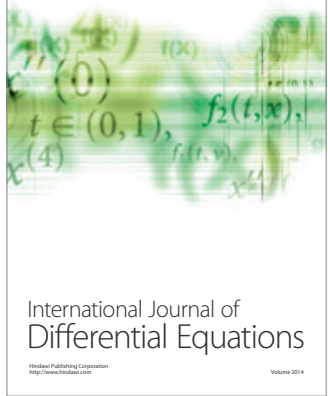
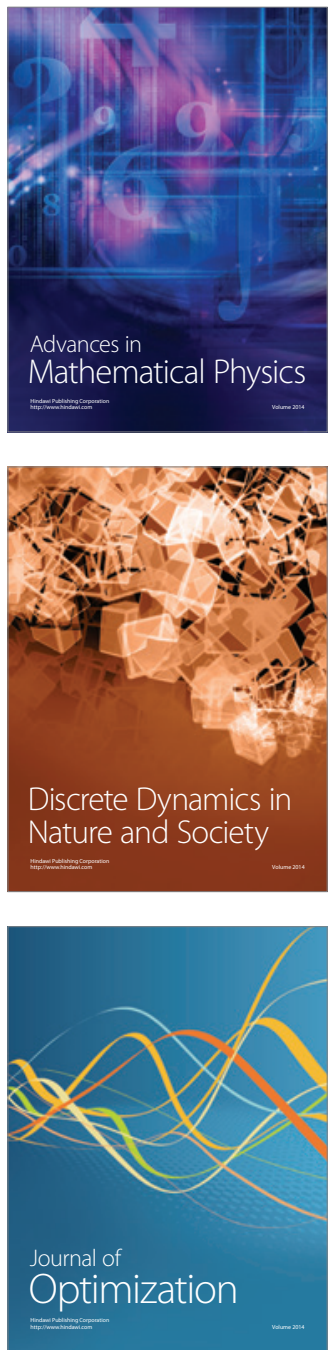